\title{
A modified Mann iterative scheme based on the generalized explicit methods for quasi-nonexpansive mappings in Banach spaces
}

\author{
Thierno M.M. Sow ${ }^{a}$ \\ ${ }^{a}$ Department of Mathematics, Gaston Berger University, \\ Saint-Louis Senegal.
}

\begin{abstract}
In this paper, we introduce and study a new iterative algorithm which is a combination of a modified Mann iterative scheme and a generalized explicit methods (GEM) for finding a common fixed points of an infinite family of quasi-nonexpansive mappings in Banach spaces. Under suitable conditions, some strong convergence theorems for finding a common fixed points of an infinite family of quasi-nonexpansive mappings are obtained without imposing any compactness assumption. Presented results improve and generalize many known results in the current literature.
\end{abstract}

Keywords: Common fixed points; Quasi-nonexpansive mappings; Midpoint rule; Banach spaces. 2010 MSC: 47H09; 47H10; 47J25.

\section{Introduction}

Let $X$ be a real normed space, $K$ be a nonempty subset of $X$. A map $T: K \rightarrow X$ is said to be Lipschitz if there exists an $L \geq 0$ such that

$$
\|T x-T y\| \leq L\|x-y\| \quad \text { for all } x, y \in K
$$

if $L<1, T$ is called contraction and if $L=1, T$ is called nonexpansive.

We denote by $F(T)$ the set of fixed points of the mapping $T$, that is $F(T):=\{x \in D(T): x=T x\}$. We

Email address: sowthierno89@gmail.com (Thierno M.M. Sow )

Received April 23, 2019, 2019, Accepted: June 17, 2019, Online: June 24, 2019. 
assume that $F(T)$ is nonempty. If $T$ is nonexpansive mappings, it is well know $F(T)$ is closed and convex. A map $T$ is called quasi- nonexpansive if $\|T x-p\| \leq\|x-p\|$ holds for all $\mathrm{x}$ in $\mathrm{K}$ and $p \in F(T)$.

We note that the following inclusions hold for the classes of the mappings:

Firmly nonexpansive $\subset$ nonexpansive $\subset$ quasi-nonexpansive.

We illustrate these by the following example.

Example 1.1. Let $X=l_{\infty}$ and $C:=\left\{x \in l_{\infty}:\|x\|_{\infty} \leq 1\right\}$. Define $T: C \rightarrow C$ by $T x=\left(0, x^{2}{ }_{1}, x^{2}{ }_{2}, x^{3}{ }_{3}, \ldots\right)$ for $x=\left(x_{1}, x_{2}, x_{3}, \ldots\right)$ in $C$. Then, it is clear that $T$ is continuous and maps $C$ into $C$. Moreover, $T p=p$ if and only if $p=0$. Futhermore,

$$
\begin{aligned}
\|T x-p\|_{\infty} & =\|T x\|_{\infty}=\left\|\left(0, x^{2}{ }_{1}, x^{2}{ }_{2}, x^{2}{ }_{3}, \ldots\right)\right\|_{\infty} \\
& \leq\left\|\left(x_{1}, x_{2}, x_{3}, \ldots\right)\right\|_{\infty} \\
& =\|x-p\|_{\infty} .
\end{aligned}
$$

Therefore, $T$ is quasi-nonexpansive. However, $T$ is not nonexpansive.

Many problems arising in different areas of mathematics, such as optimization, variational analysis and differential equations, engineering and science problems can be modeled by the equation

$$
x=T x,
$$

where $T$ is a nonexpansive mapping. The solution set of this equation coincide to a fixed points set of $T$. Such operators have been studied extensively (see, e.g., Yao et al. [24], Chidume [4], Marino et al. [15] and the references therein).

Historically, one of the most investigated methods approximating fixed points of nonexpansive mappings dates back to 1953 and is known as Mann's method, in light of Mann [10]. Let $C$ be a nonempty, closed and convex subset of a Banach space X, Mann's scheme is defined by

$$
\left\{\begin{array}{l}
x_{0} \in C, \\
x_{n+1}=\alpha_{n} x_{n}+\left(1-\alpha_{n}\right) T x_{n},
\end{array}\right.
$$

$\left\{\alpha_{n}\right\}$ is a sequence in $(0,1)$. But Mann's iteration process has only weak convergence, even in Hilbert space setting. Therefore, many authors try to modify Mann's iteration to have strong convergence for nonlinear operators.

Over the last several years, the implicit midpoint rule (IMR) has become a powerful numerical method for numerically solving time-dependent differential equations (in particular, stiff equations) and differential algebraic equations (see, [18]). Consider the following initial value problem:

$$
x^{\prime}(t)=f(x(t)), x_{0}=x\left(t_{0}\right)
$$

where $f: \mathbb{R}^{M} \rightarrow \mathbb{R}^{M}$ is a continuous function. The IMR is an implicit method given by the following finite difference scheme [6]:

$$
\left\{\begin{array}{l}
y_{0}=x_{0} \\
y_{n+1}=y_{n}+h f\left(\frac{y_{n+1}+y_{n}}{2}\right),
\end{array}\right.
$$

where $h>0$ is a time step. It is known that if $f: \mathbb{R}^{M} \rightarrow \mathbb{R}^{M}$ is Lipschitz continuous and sufficiently smooth, then the sequence $\left\{y_{n}\right\}$ converges to the exact solution of (4) as $h \rightarrow 0$ uniformly over $t \in\left[t_{0}, t^{*}\right]$ for any fixed $t^{*}>0$. 
Based on IMR (5), Xu et al. [23] applied the viscosity approximation method introduced by Moudafi [13] to the IMR for a nonexpansive mapping $T$ and proposed the following viscosity implicit midpoint rule (VIMR) in Hilbert spaces $H$ as follows:

$$
x_{n+1}=\alpha_{n} f\left(x_{n}\right)+\left(1-\alpha_{n}\right) T\left(\frac{x_{n+1}+x_{n}}{2}\right), n \geq 1
$$

where $\left\{\alpha_{n}\right\}$ is a real control condition in $(0,1)$. They also proved that the sequence $\left\{x_{n}\right\}$ generated by (6) converges strongly to a point $x^{*} \in F(T)$, which solves the variational inequality

$$
\left\langle x^{*}-f\left(x^{*}\right), x^{*}-p\right\rangle \leq 0, \quad \forall p \in F(T) .
$$

Recently, Ke and Ma [25] improved the VIMR by replacing the midpoint by any point of the interval $\left[x_{n}, x_{n+1}\right]$. They constructed the so-called method generalized viscosity implicit rules (GVIR) for a nonexpansive mapping as follows:

$$
x_{n+1}=\alpha_{n} f\left(x_{n}\right)+\left(1-\alpha_{n}\right) T\left(s_{n} x_{n}+\left(1-s_{n}\right) x_{n+1}\right), \quad n \geq 1 .
$$

They showed that $\left\{x_{n}\right\}$ defined by (8) converges strongly to $x^{*} \in F(T)$, which solves the variational inequality problem (1).

In numerical analysis, it is clear that the computation by the IMR is not an easy work in practice. Because the IMR need to compute at every time steps, it can be much harder to implement. To overcome this difficulty, for solving (4), we consider the helpful method, the so-called explicit midpoint method (EMR), given by the following finite difference scheme

$$
\left\{\begin{array}{l}
y_{0}=x_{0} \\
\bar{y}_{n+1}=y_{n}+h f\left(y_{n}\right), \\
y_{n+1}=y_{n}+h f\left(\frac{\bar{y}_{n+1}+y_{n}}{2}\right) .
\end{array}\right.
$$

It is easy to see that the explicit midpoint method calculates the state of a system at the next time from the state of the system at the current time [7].

In 2017, Marino et al. [15] applied the sequence (8) and the explicit midpoint method (9) to established the following so-called general viscosity explicit rule for quasi-nonexpansive mappings $T$ in Hilbert spaces:

$$
\left\{\begin{array}{l}
\bar{x}_{n+1}=\beta_{n} x_{n}+\left(1-\beta_{n}\right) T x_{n} \\
x_{n+1}=\alpha_{n} f\left(x_{n}\right)+\left(1-\alpha_{n}\right) T\left(s_{n} x_{n}+\left(1-s_{n}\right) \bar{x}_{n+1}\right)
\end{array}\right.
$$

where $\mathrm{f}$ is a contraction and $\left\{\alpha_{n}\right\},\left\{\beta_{n}\right\}$, and $\left\{s_{n}\right\}$ are the sequences in $(0,1)$. They proved, under suitable conditions on the sequence parameters, that the generalized viscosity explicit rule 10 strongly converges to the set of $F(T)$, which is also the solution of the variational inequality problem (1).

Recently, Sow et al.[19] motivated by the fact that Mann algorithm method is remarkably useful for finding fixed points of nonexpansive mapping, proved the following theorem.

Theorem 1.2 (Sow et al. [19]). Let $E$ be a uniformly smooth real Banach space having a weakly continuous duality map and $K$ a nonempty, closed and convex cone of $E$. Let $T: K \rightarrow K$ be a nonexpansive mapping with $F(T) \neq \emptyset$. Let $\left\{\lambda_{n}\right\}$ and $\left\{\alpha_{n}\right\}$ be two sequences in $(0,1)$. Let $\left\{x_{n}\right\}$ be a sequence defined iteratively from arbitrary $x_{0} \in K$ by:

$$
x_{n+1}=\alpha_{n}\left(\lambda_{n} x_{n}\right)+\left(1-\alpha_{n}\right) T x_{n} .
$$

Suppose the following conditions hold:

(i) $\lim _{n \rightarrow \infty} \alpha_{n}=0 ; \quad$ (ii) $\sum_{n=0}^{\infty} \alpha_{n}=\infty$ and $\sum_{n=0}^{\infty}\left|\alpha_{n}-\alpha_{n+1}\right|<\infty$ 
(iii) $\lim _{n \rightarrow \infty} \lambda_{n}=1, \sum_{n=0}^{\infty}\left(1-\lambda_{n}\right) \alpha_{n}=\infty$, and $\sum_{n=0}^{\infty}\left|\lambda_{n}-\lambda_{n+1}\right|<\infty$

Then, the sequence $\left\{x_{n}\right\}$ generated by (11) converges strongly to $x^{*} \in F(T)$.

Motivated and inspired by the above works, the purpose of the paper is to construct an iterative algorithm based on a modified Mann iterative scheme and prove strong convergence theorems for finding a common fixed points of an infinite family of quasi-nonexpansive mappings in real Banach spaces having a weakly continuous duality maps. No compactness assumption is made. The algorithm and results presented in this paper improve and extend some recents results. Finally, our method of proof is of independent interest.

\section{Preliminaries}

Let $E$ be a Banach space with norm $\|\cdot\|$ and dual $E^{*}$. For any $x \in E$ and $x^{*} \in E^{*},\left\langle x^{*}, x\right\rangle$ is used to refer to $x^{*}(x)$. Let $\varphi:[0,+\infty) \rightarrow[0, \infty)$ be a strictly increasing continuous function such that $\varphi(0)=0$ and $\varphi(t) \rightarrow+\infty$ as $t \rightarrow \infty$. Such a function $\varphi$ is called gauge. Associed to a gauge a duality map $J_{\varphi}: E \rightarrow 2^{E^{*}}$ defined by:

$$
J_{\varphi}(x):=\left\{x^{*} \in E^{*}:\left\langle x, x^{*}\right\rangle=\|x\| \varphi(\|x\|),\left\|x^{*}\right\|=\varphi(\|x\|)\right\}, x \in E .
$$

If the gauge is defined by $\varphi(t)=t$, then the corresponding duality map is called the normalized duality map and is denoted by $J$. Hence the normalized duality map is given by

$$
J(x):=\left\{x^{*} \in E^{*}:\left\langle x, x^{*}\right\rangle=\|x\|^{2}=\left\|x^{*}\right\|^{2}=\right\}, \forall x \in E .
$$

Notice that

$$
J_{\varphi}(x)=\frac{\varphi(\|x\|)}{\|x\|} J(x), x \neq 0 .
$$

Let $E$ be a real normed space and let $S:=\{x \in E:\|x\|=1\}$. E is said to be smooth if the limit

$$
\lim _{t \rightarrow 0^{+}} \frac{\|x+t y\|-\|x\|}{t}
$$

exists for each $x, y \in S$. It is known that $E$ is smooth if and only if each duality map $J_{\varphi}$ is single-valued, that $E$ is Frechet differentiable if and only if each duality map $J_{\varphi}$ is norm-to-norm continuous in $E$, and that $E$ is uniformly smooth if and only if each duality map $J_{\varphi}$ is norm-to-norm uniformly continuous on bounded subsets of $E$.

Following Browder [2, we say that a Banach space has a weakly continuous duality map if there exists a gauge $\varphi$ such that $J_{\varphi}$ is single-valued and is weak-to-weak* sequentially continuous, i.e., if $\left(x_{n}\right) \subset E, x_{n} \stackrel{w}{\rightarrow} x$, then $J_{\varphi}\left(x_{n}\right) \stackrel{w^{*}}{\longrightarrow} J_{\varphi}(x)$. It is know that $l^{p}(1<p<\infty)$ has a weakly continuous duality map with gauge $\varphi(t)=t^{p-1}$. (see [3] fore more details on duality maps). Finally recall that a Banach space $E$ satisfies Opial property (see, e.g., [15]) if $\limsup \left\|x_{n}-x\right\|<\limsup \left\|x_{n}-y\right\|$ whenever $x_{n} \stackrel{w}{\rightarrow} x, x \neq y$. A Banach space $E$ that has a weakly continuous duality map satisfies Opial's property.

The following lemma can be found in [5].

Lemma 2.1 (Demiclosedness principle [5]). Let $E$ be a reflexive Banach space satisfying Opial's property, $K$ be a closed convex subset of $E$, and $T: K \rightarrow K$ be a nonexpansive mapping. Then $I-T$ is demiclosed; that is,

$$
\left\{x_{n}\right\} \subset K, x_{n} \rightarrow x \in K \text { and }(I-T) x_{n} \rightarrow y \text { implies that }(I-T) x=y .
$$


Lemma $2.2([9])$. Assume that a Banach space E has a weakly continous duality mapping $J_{\varphi}$ with jauge $\varphi$.

$$
\Phi(\|x+y\|) \leq \Phi(\|x\|)+\left\langle y, J_{\varphi}(x+y)\right\rangle
$$

for all $x, y \in E$.

In particular, for all $x, y \in E$,

$$
\|x+y\|^{2} \leq\|x\|^{2}+2\langle y, J(x+y)\rangle .
$$

Lemma 2.3. (Xu,[21], Zalinescu [25]) Let $E$ be a uniformly convex real Banach space. For arbitrary $r>0$, let $B_{r}:=\{x \in E:\|x\| \leq r\}$ and $\beta_{n} \in[0,1]$. Then there exists a continuous, strictly increasing and convex function

$$
g:[0,2 r] \rightarrow \mathbb{R}^{+}, g(0)=0
$$

such that for all $x, y \in B_{r}$

$$
\left\|\beta_{n} x+\left(1-\beta_{n}\right) y\right\|^{2} \leq \beta_{n}\|x\|^{2}+\left(1-\beta_{n}\right)\|y\|^{2}-\left(1-\beta_{n}\right) \beta_{n} g(\|x-y\|) .
$$

Lemma $2.4(\mathrm{Xu}, 22])$. Assume that $\left\{a_{n}\right\}$ is a sequence of nonnegative real numbers such that $a_{n+1} \leq$ $\left(1-\alpha_{n}\right) a_{n}+\alpha_{n} \sigma_{n}$ for all $n \geq 0$, where $\left\{\alpha_{n}\right\}$ is a sequence in $(0,1)$ and $\left\{\sigma_{n}\right\}$ is a sequence in $\mathbb{R}$ such that (a) $\sum_{n=0}^{\infty} \alpha_{n}=\infty$, (b) $\limsup _{n \rightarrow \infty} \sigma_{n} \leq 0$ or $\sum_{n=0}^{\infty}\left|\sigma_{n} \alpha_{n}\right|<\infty$. Then $\lim _{n \rightarrow \infty} a_{n}=0$.

Lemma 2.5. [11] Let $t_{n}$ be a sequence of real numbers that does not decrease at infinity in a sense that there exists a subsequence $t_{n_{i}}$ of $t_{n}$ such that $t_{n_{i}}$ such that $t_{n_{i}} \leq t_{n_{i+1}}$ for all $i \geq 0$. For sufficiently large numbers $n \in \mathbb{N}$, an integer sequence $\{\tau(n)\}$ is defined as follows:

$$
\tau(n)=\max \left\{k \leq n: t_{k} \leq t_{k+1}\right\} .
$$

Then, $\tau(n) \rightarrow \infty$ as $n \rightarrow \infty$ and

$$
\max \left\{t_{\tau(n)}, t_{n}\right\} \leq t_{\tau(n)+1} .
$$

Lemma 2.6 (Aoyama et. al [1, Nilsrakoo et al. [14]). Let $K$ be a nonempty closed subset of a Banach space and let $\left\{T_{n}\right\}_{n \geq 0}$ be a sequence of mappings of $K$ into itself. Suppose that $\sum_{n=0}^{\infty} \sup \left\{\left\|T_{n+1} x-T_{n} x\right\|: x \in\right.$ $B\}<\infty$ for any bounded subset $B$ of $K$. Then, for any $x \in K\left\{T_{n} x\right\}$ converges strongly to some point of $K$. Moreover, let $T$ be a mapping of $K$ into itself defined by $T x=\lim _{n \rightarrow \infty} T_{n} x$ for all $x \in K$. Then,

$$
\lim _{n \rightarrow \infty} \sup _{x \in K}\left\|T_{n} x-T x\right\|=0 .
$$

Let $C$ be a nonempty subsets of real Banach space $E$. A mapping $Q_{C}: E \rightarrow C$ is said to be sunny if

$$
Q_{C}\left(Q_{C} x+t\left(x-Q_{C} x\right)\right)=Q_{C} x
$$

for each $x \in E$ and $t \geq 0$. A mapping $Q_{C}: E \rightarrow C$ is said to be a retraction if $Q_{C} x=x$ for each $x \in C$.

Lemma 2.7. [17] Let $C$ and $D$ be nonempty subsets of a real Banach space $E$ with $D \subset C$ and $Q_{D}: C \rightarrow D$ a retraction from $C$ into $D$. Then $Q_{D}$ is sunny and nonexpansive if and only if

$$
\left\langle z-Q_{D} z, j\left(y-Q_{D} z\right)\right\rangle \leq 0
$$

for all $z \in C$ and $y \in D$.

It is noted that Lemma 2.7 still holds if the normalized duality map is replaced by the general duality map $J_{\varphi}$, where $\varphi$ is gauge function.

Remark 2.8. If $K$ is a nonempty closed convex subset of a Hilbert space $H$, then the nearest point projection $P_{K}$ from $H$ to $K$ is the sunny nonexpansive retraction. 


\section{Mains results}

Throughout this section, we assume that $E$ be a uniformly convex real Banach space having a weakly continuous duality map $J_{\varphi}$ and $K$ be a nonempty, closed and convex cone of $E$. For each $n=0,1, \ldots$, let $T_{n}: K \rightarrow K$ be a quasi-nonexpansive mapping such that $F:=\bigcap_{n=0}^{\infty} F\left(T_{n}\right) \neq \emptyset$.

Now, we present the following iterative algorithm.

Algorithm 3.1. Let $\left\{x_{n}\right\}$ be a sequence defined iteratively from arbitrary $x_{0} \in K$ by:

$$
\left\{\begin{array}{l}
\bar{x}_{n+1}=\beta_{n} x_{n}+\left(1-\beta_{n}\right) T_{n} x_{n}, \\
x_{n+1}=\alpha_{n}\left(\lambda_{n} x_{n}\right)+\left(1-\alpha_{n}\right) T_{n}\left(s_{n} x_{n}+\left(1-s_{n}\right) \bar{x}_{n+1}\right),
\end{array}\right.
$$

$\left\{\beta_{n}\right\},\left\{\lambda_{n}\right\},\left\{s_{n}\right\}$ and $\left\{\alpha_{n}\right\}$ be sequences in $(0,1)$ satisfying:

(i) $\lim _{n \rightarrow \infty} \alpha_{n}=0 ; \quad$ (ii) $\lim _{n \rightarrow \infty} \inf \left(1-s_{n}\right) \beta_{n}\left(1-\beta_{n}\right)>0$.

(iii) $\lim _{n \rightarrow \infty} \lambda_{n}=1$ and $\sum_{n=0}^{\infty}\left(1-\lambda_{n}\right) \alpha_{n}=\infty$.

For strong convergence of our Algorithm, we assume the following assumptions.

Assumption 3.2. (a) $\sum_{n=0}^{\infty} \sup \left\{\left\|T_{n+1} x-T_{n} x\right\|: \quad x \in B\right\}<\infty$ for any bounded subset $B$ of $K$ and $F(T)=$ $\bigcap_{n=0}^{\infty} F\left(T_{n}\right)$ where $T$ be a mapping of $K$ into itself defined by $T x=\lim _{n \rightarrow \infty} T_{n} x$ for all $x \in K$.

(b) $I-T$ is demiclosed at the origin.

We now prove the following results.

Theorem 3.3. Let Assumptions 3.2 hold. Then, the sequence $\left\{x_{n}\right\}$ generated by (13) converges strongly to $x^{*} \in F$ where $x^{*}=Q_{F}(0)$ with $Q_{F}$ the sunny nonexpansive retraction of $K$ onto $F$.

Proof. For each $n \geq 0$, we put $z_{n}:=s_{n} x_{n}+\left(1-s_{n}\right) \bar{x}_{n+1}$. Let $p \in F$, from (13) and $T_{n}$ is quasi-nonexpansive, we have

$$
\begin{aligned}
\left\|z_{n}-p\right\| & =\left\|s_{n} x_{n}+\left(1-s_{n}\right) \bar{x}_{n+1}-p\right\| \\
& \leq s_{n}\left\|x_{n}-p\right\|+\left(1-s_{n}\right)\left\|\bar{x}_{n+1}-p\right\| \\
& \leq s_{n}\left\|x_{n}-p\right\|+\left(1-s_{n}\right)\left\|\beta_{n} x_{n}+\left(1-\beta_{n}\right) T_{n} x_{n}-p\right\| \\
& \leq s_{n}\left\|x_{n}-p\right\|+\left(1-s_{n}\right)\left[\beta_{n}\left\|x_{n}-p\right\|+\left(1-\beta_{n}\right)\left\|T_{n} x_{n}-p\right\|\right]
\end{aligned}
$$

Hence,

$$
\left\|z_{n}-p\right\| \leq\left\|x_{n}-p\right\| .
$$

Now, we prove that the sequence $\left\{x_{n}\right\}$ is bounded. Let $p \in F$.

Using (13) and inequality (14), we have

$$
\begin{aligned}
\left\|x_{n+1}-p\right\| & =\left\|\alpha_{n}\left(\lambda_{n} x_{n}\right)+\left(1-\alpha_{n}\right) T_{n} z_{n}-p\right\| \\
& \leq \alpha_{n} \lambda_{n}\left\|x_{n}-p\right\|+\left(1-\alpha_{n}\right)\left\|T_{n} z_{n}-p\right\|+\left(1-\lambda_{n}\right) \alpha_{n}\|p\| \\
& \leq \alpha_{n} \lambda_{n}\left\|x_{n}-p\right\|+\left(1-\alpha_{n}\right)\left\|x_{n}-p\right\|+\left(1-\lambda_{n}\right) \alpha_{n}\|p\| \\
& \leq\left[1-\left(1-\lambda_{n}\right) \alpha_{n}\right]\left\|x_{n}-p\right\|+\left(1-\lambda_{n}\right) \alpha_{n}\|p\| \\
& \leq \max \left\{\left\|x_{n}-p\right\|,\|p\|\right\} .
\end{aligned}
$$


By induction, it is easy to see that

$$
\left\|x_{n}-p\right\| \leq \max \left\{\left\|x_{0}-p\right\|,\|p\|\right\}, \quad n \geq 1 .
$$

Hence $\left\{x_{n}\right\}$ is bounded also are $\left\{\bar{x}_{n}\right\}$, and $\left\{T_{n} x_{n}\right\}$.

Using Lemma 2.3, convexity of $\|\cdot\|^{2}$ and 13 , we have

$$
\begin{aligned}
\left\|T_{n} z_{n}-p\right\|^{2} \leq & \left\|z_{n}-p\right\|^{2} \\
= & \left\|s_{n} x_{n}+\left(1-s_{n}\right) \bar{x}_{n+1}-p\right\|^{2} \\
\leq & s_{n}\left\|x_{n}-p\right\|^{2}+\left(1-s_{n}\right)\left\|\bar{x}_{n+1}-p\right\|^{2} \\
\leq & s_{n}\left\|x_{n}-p\right\|^{2}+\left(1-s_{n}\right)\left\|\beta_{n} x_{n}+\left(1-\beta_{n}\right) T_{n} x_{n}-p\right\|^{2} \\
\leq & s_{n}\left\|x_{n}-p\right\|^{2}+\left(1-s_{n}\right)\left[\beta_{n}\left\|x_{n}-p\right\|^{2}+\left(1-\beta_{n}\right)\left\|T_{n} x_{n}-p\right\|^{2}\right. \\
& \left.-\beta_{n}\left(1-\beta_{n}\right) g\left(\left\|x_{n}-T_{n} x_{n}\right\|\right)\right] \\
\leq & \left\|x_{n}-p\right\|^{2}-\left(1-s_{n}\right) \beta_{n}\left(1-\beta_{n}\right) g\left(\left\|x_{n}-T_{n} x_{n}\right\|\right)
\end{aligned}
$$

Hence,

$$
\left\|T_{n} z_{n}-p\right\|^{2} \leq\left\|x_{n}-p\right\|^{2}-\beta_{n}\left(1-\beta_{n}\right) g\left(\left\|x_{n}-T_{n} x_{n}\right\|\right) .
$$

Therefore, by Lemma 2.2 and inequality (15), we have

$$
\begin{aligned}
\left\|x_{n+1}-p\right\|^{2}= & \left\|\alpha_{n}\left(\lambda_{n} x_{n}\right)+\left(1-\alpha_{n}\right) T_{n} z_{n}-p\right\|^{2} \\
= & \left\|\alpha_{n} \lambda_{n}\left(x_{n}-p\right)+\left(1-\alpha_{n}\right)\left(T_{n} z_{n}-p\right)-\left(1-\lambda_{n}\right) \alpha_{n} p\right\|^{2} \\
\leq & \left\|\alpha_{n}\left(\lambda_{n} x_{n}-\lambda_{n} p\right)+\left(1-\alpha_{n}\right)\left(T_{n} z_{n}-p\right)\right\|^{2}+2\left(1-\lambda_{n}\right) \alpha_{n}\left\langle p, J\left(p-x_{n+1}\right)\right\rangle \\
\leq & \alpha_{n} \lambda_{n}^{2}\left\|x_{n}-p\right\|^{2}+\left(1-\alpha_{n}\right)\left\|T_{n} z_{n}-p\right\|^{2}+2\left(1-\lambda_{n}\right) \alpha_{n}\left\langle p, J\left(p-x_{n+1}\right)\right\rangle \\
\leq & \alpha_{n} \lambda_{n}\left\|x_{n}-p\right\|^{2}+\left(1-\alpha_{n}\right)\left[\left\|x_{n}-p\right\|^{2}-\beta_{n}\left(1-\beta_{n}\right) g\left(\left\|x_{n}-T_{n} x_{n}\right\|\right)\right] \\
& +2\left(1-\lambda_{n}\right) \alpha_{n}\left\langle p, J\left(p-x_{n+1}\right)\right\rangle \\
\leq & {\left[1-\left(1-\lambda_{n}\right) \alpha_{n}\right]\left\|x_{n}-p\right\|^{2}-\left(1-\alpha_{n}\right) \beta_{n}\left(1-\beta_{n}\right) g\left(\left\|x_{n}-T_{n} x_{n}\right\|\right) } \\
& +2\left(1-\lambda_{n}\right) \alpha_{n}\left\langle p, J\left(p-x_{n+1}\right)\right\rangle .
\end{aligned}
$$

Therefore,

$$
\left(1-\alpha_{n}\right) \beta_{n}\left(1-\beta_{n}\right) g\left(\left\|x_{n}-T_{n} x_{n}\right\|\right) \leq\left\|x_{n}-p\right\|^{2}-\left\|x_{n+1}-p\right\|^{2}+2\left(1-\lambda_{n}\right) \alpha_{n}\left\langle p, J\left(p-x_{n+1}\right)\right\rangle .
$$

Since $\left\{x_{n}\right\}$ is bounded, then there exists a constant $B>0$ sucht that

$$
\left(1-\lambda_{n}\right)\left\langle p, J\left(p-x_{n+1}\right)\right\rangle \leq B, \text { for all, } n \geq 0 .
$$

Hence,

$$
\left(1-\alpha_{n}\right) \beta_{n}\left(1-\beta_{n}\right) g\left(\left\|x_{n}-T_{n} x_{n}\right\|\right) \leq\left\|x_{n}-p\right\|^{2}-\left\|x_{n+1}-p\right\|^{2}+2 \alpha_{n} B .
$$

Now we prove that $\left\{x_{n}\right\}$ converges strongly to $x^{*}$.

We divide the proof into two cases.

Case 1. Assume that the sequence $\left\{\left\|x_{n}-p\right\|\right\}$ is monotonically decreasing sequence. Then $\left\{\left\|x_{n}-p\right\|\right\}$ is convergent. Clearly, we have

$$
\left\|x_{n}-p\right\|^{2}-\left\|x_{n+1}-p\right\|^{2} \rightarrow 0 .
$$

It then implies from (17) that

$$
\lim _{n \rightarrow \infty}\left(1-\alpha_{n}\right) \beta_{n}\left(1-\beta_{n}\right) g\left(\left\|x_{n}-T_{n} x_{n}\right\|\right)=0 .
$$


Using the fact that $\lim _{n \rightarrow \infty} \inf \left(1-s_{n}\right) \beta_{n}\left(1-\beta_{n}\right)>0$ and property of $g$, we have

$$
\lim _{n \rightarrow \infty}\left\|x_{n}-T_{n} x_{n}\right\|=0
$$

We observe that,

$$
\left\|x_{n}-T x_{n}\right\| \leq\left\|x_{n}-T_{n} x_{n}\right\|+\left\|T_{n} x_{n}-T x_{n}\right\| .
$$

By inequalities (19), 20) and Lemma 2.6, we have

$$
\lim _{n \rightarrow \infty}\left\|x_{n}-T x_{n}\right\|=0 .
$$

Next, we prove that $\limsup _{n \rightarrow+\infty}\left\langle x^{*}, J_{\varphi}\left(x^{*}-x_{n}\right)\right\rangle$. Since $E$ is reflexive and $\left\{x_{n}\right\}$ is bounded, there exists a subsequence $\left\{x_{n_{k}}\right\}$ of $\left\{x_{n}\right\}$ such that $x_{n_{k}}$ converges weakly to $a$ in $K$ and

$$
\limsup _{n \rightarrow+\infty}\left\langle x^{*}, J_{\varphi}\left(x^{*}-x_{n}\right)\right\rangle=\lim _{k \rightarrow+\infty}\left\langle x^{*}, J_{\varphi}\left(x^{*}-x_{n_{k}}\right)\right\rangle .
$$

From (21) and $I-T$ is demiclosed, we obtain $a \in F$. On other hand, the assumption that the duality mapping $J_{\varphi}$ is weakly continuous, the fact that $x^{*}=Q_{F}(0)$ and Lemma 2.7, we then have

$$
\begin{aligned}
\limsup _{n \rightarrow+\infty}\left\langle x^{*}, J_{\varphi}\left(x^{*}-x_{n}\right)\right\rangle & =\lim _{k \rightarrow+\infty}\left\langle x^{*}, J_{\varphi}\left(x^{*}-x_{n_{k}}\right)\right\rangle \\
& =\left\langle x^{*}, J_{\varphi}\left(x^{*}-a\right)\right\rangle \leq 0 .
\end{aligned}
$$

Finally, we show that $x_{n} \rightarrow x^{*}$. In fact, since $\Phi(t)=\int_{0}^{t} \varphi(\sigma) d \sigma, \forall t \geq 0$, and $\varphi$ is a gauge function, then for $1 \geq k \geq 0, \Phi(k t) \leq k \Phi(t)$. From (13) and Lemma 2.2, we get that

$$
\begin{aligned}
\Phi\left(\left\|x_{n+1}-x^{*}\right\|\right) & =\Phi\left(\left\|\alpha_{n}\left(\lambda_{n} x_{n}\right)+\left(1-\alpha_{n}\right) T_{n} z_{n}-x^{*}\right\|\right) \\
& \leq \Phi\left(\left\|\alpha_{n} \lambda_{n}\left(x_{n}-x^{*}\right)+\left(1-\alpha_{n}\right)\left(T_{n} z_{n}-x^{*}\right)\right\|\right)+\left(1-\lambda_{n}\right) \alpha_{n}\left\langle x^{*}, J_{\varphi}\left(x^{*}-x_{n+1}\right)\right\rangle \\
\leq & \leq\left(\alpha_{n} \lambda_{n}\left\|x_{n}-x^{*}\right\|+\left\|\left(1-\alpha_{n}\right)\left(T_{n} z_{n}-x^{*}\right)\right\|\right)+\left(1-\lambda_{n}\right) \alpha_{n}\left\langle x^{*}, J_{\varphi}\left(x^{*}-x_{n+1}\right)\right\rangle \\
\leq & \Phi\left(\alpha_{n} \lambda_{n}\left\|x_{n}-x^{*}\right\|+\left(1-\alpha_{n}\right)\left\|x_{n}-x^{*}\right\|\right)+\left(1-\lambda_{n}\right) \alpha_{n}\left\langle x^{*}, J_{\varphi}\left(x^{*}-x_{n+1}\right)\right\rangle \\
& \leq \Phi\left(\left(1-\left(1-\lambda_{n}\right) \alpha_{n}\right)\left\|x_{n}-x^{*}\right\|\right)+\left(1-\lambda_{n}\right) \alpha_{n}\left\langle x^{*}, J_{\varphi}\left(x^{*}-x_{n+1}\right)\right\rangle \\
& \leq\left[1-\left(1-\lambda_{n}\right) \alpha_{n}\right] \Phi\left(\left\|x_{n}-x^{*}\right\|\right)+\left(1-\lambda_{n}\right) \alpha_{n}\left\langle x^{*}, J_{\varphi}\left(x^{*}-x_{n+1}\right)\right\rangle .
\end{aligned}
$$

From Lemma 2.4, its follows that $x_{n} \rightarrow x^{*}$.

Case 2. Assume that the sequence $\left\{\left\|x_{n}-x^{*}\right\|\right\}$ is not monotonically decreasing sequence. Set $B_{n}=\left\|x_{n}-x^{*}\right\|$ and $\tau: \mathbb{N} \rightarrow \mathbb{N}$ be a mapping for all $n \geq n_{0}$ (for some $n_{0}$ large enough) by $\tau(n)=\max \left\{k \in \mathbb{N}: k \leq n, B_{k} \leq\right.$ $\left.B_{k+1}\right\}$.

We have $\tau$ is a non-decreasing sequence such that $\tau(n) \rightarrow \infty$ as $n \rightarrow \infty$ and $B_{\tau(n)} \leq B_{\tau(n)+1}$ for $n \geq n_{0}$. From (17), we have

$$
\left(1-\alpha_{\tau(n)}\right) \beta_{\tau(n)}\left(1-\beta_{\tau(n)}\right) g\left(\left\|x_{\tau(n)}-T_{\tau(n)} x_{\tau(n)}\right\|\right) \leq 2 \alpha_{\tau(n)} B \rightarrow 0 \text { as } n \rightarrow \infty .
$$

Hence,

$$
\lim _{n \rightarrow \infty}\left\|x_{\tau(n)}-T_{\tau(n)} x_{\tau(n)}\right\|=0 .
$$

At the same time, we observe that

$$
\left\|x_{\tau(n)}-T x_{\tau(n)}\right\| \leq\left\|x_{\tau(n)}-T_{\tau(n)} x_{\tau(n)}\right\|+\left\|T_{\tau(n)} x_{\tau(n)}-T x_{\tau(n)}\right\| .
$$

Thanks inequalities 222, 23) and Lemma 2.6, we have

$$
\lim _{n \rightarrow \infty}\left\|x_{\tau(n)}-T x_{\tau(n)}\right\|=0 .
$$


By same argument as in case 1 , we can show that $x_{\tau(n)}$ converges weakly in $E$ and $\limsup _{n \rightarrow+\infty}\left\langle x^{*}, J_{\varphi}\left(x^{*}-x_{\tau(n)}\right)\right\rangle \leq$ 0 . We have for all $n \geq n_{0}$,

$$
0 \leq \Phi\left(\left\|x_{\tau(n)+1}-x^{*}\right\|\right)-\Phi\left(\left\|x_{\tau(n)}-x^{*}\right\|\right) \leq\left(1-\lambda_{\tau(n)}\right) \alpha_{\tau(n)}\left[-\Phi\left(\left\|x_{\tau(n)}-x^{*}\right\|\right)+\left\langle x^{*}, J_{\varphi}\left(x^{*}-x_{\tau(n)+1}\right)\right\rangle\right],
$$

which implies that

$$
\Phi\left(\left\|x_{\tau(n)}-x^{*}\right\|\right) \leq\left\langle x^{*}, J_{\varphi}\left(x^{*}-x_{\tau(n)+1}\right)\right\rangle .
$$

Then, we have

$$
\lim _{n \rightarrow \infty} \Phi\left(\left\|x_{\tau(n)}-x^{*}\right\|\right)=0
$$

Therefore,

$$
\lim _{n \rightarrow \infty} B_{\tau(n)}=\lim _{n \rightarrow \infty} B_{\tau(n)+1}=0 .
$$

Thus, by Lemma 2.5, we conclude that

$$
0 \leq B_{n} \leq \max \left\{B_{\tau(n)}, B_{\tau(n)+1}\right\}=B_{\tau(n)+1} .
$$

Hence, $\lim _{n \rightarrow \infty} B_{n}=0$, that is $\left\{x_{n}\right\}$ converges strongly to $x^{*}$. This completes the proof.

Remark 3.4. let $\left\{T_{n}\right\}_{n \geq 0}$ be a sequence of nonexpansive mappings of $K$ into $K$, let $\left\{\lambda_{n}\right\}_{n \geq 0}$ be a sequence of real number such that and $0 \leq \lambda_{n} \leq 1$. For each $n \geq 0$, we define a mapping $W_{n}$ of $K$ into $K$ as follows:

$$
\begin{aligned}
& U_{n, n+1}=I, \\
& U_{n, n}=\lambda_{n} T_{n} U_{n, n+1}+\left(1-\lambda_{n}\right) I, \\
& U_{n, n-1}=\lambda_{n-1} T_{n-1} U_{n, n}+\left(1-\lambda_{n-1}\right) I, \\
& \vdots \\
& U_{n, k}=\lambda_{k} T_{k} U_{n, k+1}+\left(1-\lambda_{k}\right) I, \\
& \vdots \\
& U_{n, 2}=\lambda_{2} T_{2} U_{n, 3}+\left(1-\lambda_{2}\right) I, \\
& W_{n}=U_{n, 1}=\lambda_{1} T_{1} U_{n, 2}+\left(1-\lambda_{1}\right) I .
\end{aligned}
$$

Such that is called $W_{n}$ is the so called $W$-mapping generated by an countable infinite family of nonexpansive mappings $T_{1}, T_{2}, \ldots, T_{n}, \ldots$ and scalars $\lambda_{1}, \lambda_{2}, \ldots, \lambda_{n}, \ldots$ such that the common fixed points set $F:=\bigcap_{n=1}^{\infty} F\left(T_{n}\right) \neq$ $\emptyset$, see for example [20]. Clearly, $W_{n}$ is nonexpansive and from [20], we know that $\bigcap_{n=1}^{\infty} F\left(T_{n}\right)=F\left(W_{n}\right)$. Furthermore, from [16], we have the sequence $\left\{W_{n}\right\}_{n \geq 1}$ satisfies the condition $\sum_{n=0}^{\infty} \sup \left\{\left\|W_{n+1} x-W_{n} x\right\|\right.$ : $x \in B\}<\infty$ for any bounded subset $B$ of $K$ imposed in Theorem 3.3 .

By above remark, Lemma 2.1 and the fact that nonexpansive mapping is quasi-nonexpansive. We obtain the following results.

Theorem 3.5. Let $E$ be a uniformly convex real Banach space having a weakly continuous duality map $J_{\varphi}$ and $K$ be a nonempty, closed and convex cone of $E$. For each $n=0,1, \ldots$, let $T_{n}: K \rightarrow K$ be a nonexpansive mapping such that $F:=\bigcap_{n=0}^{\infty} F\left(T_{n}\right) \neq \emptyset$. Let $\left\{x_{n}\right\}$ be a sequence defined iteratively from arbitrary $x_{0} \in K$ by:

$$
\left\{\begin{array}{l}
\bar{x}_{n+1}=\beta_{n} x_{n}+\left(1-\beta_{n}\right) W_{n} x_{n}, \\
x_{n+1}=\alpha_{n}\left(\lambda_{n} x_{n}\right)+\left(1-\alpha_{n}\right) W_{n}\left(s_{n} x_{n}+\left(1-s_{n}\right) \bar{x}_{n+1}\right)
\end{array}\right.
$$


$\left\{\beta_{n}\right\},\left\{\lambda_{n}\right\},\left\{s_{n}\right\}$ and $\left\{\alpha_{n}\right\}$ be a sequences in $(0,1)$ satisfying:

(i) $\lim _{n \rightarrow \infty} \alpha_{n}=0 ; \quad$ (ii) $\lim _{n \rightarrow \infty} \inf \left(1-s_{n}\right) \beta_{n}\left(1-\beta_{n}\right)>0$.

(iii) $\lim _{n \rightarrow \infty} \lambda_{n}=1$ and $\sum_{n=0}^{\infty}\left(1-\lambda_{n}\right) \alpha_{n}=\infty$.

Then, the sequence $\left\{x_{n}\right\}$ generated by (25) converges strongly to $x^{*} \in F$ where $x^{*}=Q_{F}(0)$ with $Q_{F}$ the sunny nonexpansive retraction of $K$ onto $F$.

We apply Theorem 3.3 to approximate fixed points of quasi-nonexpansive mappings.

Corollary 3.6. Let $E$ be a uniformly convex real Banach space having a weakly continuous duality map $J_{\varphi}$ and $K$ be a nonempty, closed and convex cone of $E$. Let $T: K \rightarrow K$ be a quasi-nonexpansive mapping such that $F(T) \neq \emptyset$. Let $\left\{x_{n}\right\}$ be a sequence defined iteratively from arbitrary $x_{0} \in K$ by:

$$
\left\{\begin{array}{l}
\bar{x}_{n+1}=\beta_{n} x_{n}+\left(1-\beta_{n}\right) T x_{n}, \\
x_{n+1}=\alpha_{n}\left(\lambda_{n} x_{n}\right)+\left(1-\alpha_{n}\right) T\left(s_{n} x_{n}+\left(1-s_{n}\right) \bar{x}_{n+1}\right)
\end{array}\right.
$$

$\left\{\beta_{n}\right\},\left\{\lambda_{n}\right\},\left\{s_{n}\right\}$ and $\left\{\alpha_{n}\right\}$ be sequences in $(0,1)$ satisfying:

(i) $\lim _{n \rightarrow \infty} \alpha_{n}=0 ; \quad$ (ii) $\lim _{n \rightarrow \infty} \inf \left(1-s_{n}\right) \beta_{n}\left(1-\beta_{n}\right)>0$.

(iii) $\lim _{n \rightarrow \infty} \lambda_{n}=1$ and $\sum_{n=0}^{\infty}\left(1-\lambda_{n}\right) \alpha_{n}=\infty$.

Assume that $I-T$ is demiclosed at the origin. Then, the sequence $\left\{x_{n}\right\}$ generated by (26) converges strongly to $x^{*} \in F(T)$ where $x^{*}=Q_{F(T)}(0)$ with $Q_{F(T)}$ the sunny nonexpansive retraction of $K$ onto $F(T)$.

Remark 3.7. In our theorems, we assume that $K$ is a cone. But, in some cases, for example, if $K$ is the closed unit ball, we can weaken this assumption to the following: $\lambda x \in K$ for all $\lambda \in(0,1)$ and $x \in K$. Therefore, in the case where $E$ is a real Hilbert space or $E=l_{p}, 1<p<\infty$, our results can be used to approximate fixed points of an infinite family of quasi-nonexpansive mappings from the closed unit ball to itself.

Corollary 3.8. Assume that $E=l_{p}, 1<p<\infty$ or $E$ is a real Hilbert space. Let $B$ be the closed unit ball of E. For each $n=0,1, \ldots$, let $T_{n}: B \rightarrow B$ be a quasi-nonexpansive mapping such that $F:=\bigcap_{n=0}^{\infty} F\left(T_{n}\right) \neq \emptyset$.

Let $\left\{x_{n}\right\}$ be a sequence defined iteratively from arbitrary $x_{0} \in B$ by:

$$
\left\{\begin{array}{l}
\bar{x}_{n+1}=\beta_{n} x_{n}+\left(1-\beta_{n}\right) T_{n} x_{n}, \\
x_{n+1}=\alpha_{n}\left(\lambda_{n} x_{n}\right)+\left(1-\alpha_{n}\right) T_{n}\left(s_{n} x_{n}+\left(1-s_{n}\right) \bar{x}_{n+1}\right)
\end{array}\right.
$$

$\left\{\beta_{n}\right\},\left\{\lambda_{n}\right\},\left\{s_{n}\right\}$ and $\left\{\alpha_{n}\right\}$ be sequences in $(0,1)$ satisfying:

(i) $\lim _{n \rightarrow \infty} \alpha_{n}=0 ; \quad$ (ii) $\lim _{n \rightarrow \infty} \inf \left(1-s_{n}\right) \beta_{n}\left(1-\beta_{n}\right)>0$.

(iii) $\lim _{n \rightarrow \infty} \lambda_{n}=1$ and $\sum_{n=0}^{\infty}\left(1-\lambda_{n}\right) \alpha_{n}=\infty$.

Let Assumptions 3.2 hold. Then, the sequence $\left\{x_{n}\right\}$ generated by (27) converges strongly to $x^{*} \in F$ where $x^{*}=Q_{F}(0)$ with $Q_{F}$ the sunny nonexpansive retraction of $K$ onto $F$.

Now, we give some remarks on our results as follows:

(1) The proof methods of our results are very different from the ones of sow et al.[19] (see, Corollary 3.6) and we extend results of Sow et al. from nonexpensive mappings to quasi-nonexpansive mappings. Further, we 
remove the following conditions: $\sum_{n=0}^{\infty}\left|\alpha_{n}-\alpha_{n+1}\right|<\infty$ and $\sum_{n=0}^{\infty}\left|\lambda_{n}-\lambda_{n+1}\right|<\infty, \quad \sum_{n=0}^{\infty} \alpha_{n}=\infty$, in Theorem 1.2 of [19] (see, Corollary 3.6).

(2) Our results improve many recent results using Mann's method to approximate fixed points of nonexpansive mappings.

(3) Our results are applicable for finding minimum-norm fixed points of an infinte family of quasi-nonexpansive mappings in Hilbert spaces.

Remark 3.9. Let $\alpha_{n}=\frac{1}{10 n+1}, \beta_{n}=\frac{1}{20 n+1}+0.4, s_{n}=\frac{1}{30 n+1}+0.3$ and $\lambda_{n}=1-\frac{1}{\sqrt{n}}$. It is easy to see that the sequences $\left\{\alpha_{n}\right\},\left\{\beta_{n}\right\},\left\{s_{n}\right\}$ and $\left\{\lambda_{n}\right\}$ satisfy the conditions (i), (ii) and (iii) of Theorem 3.3.

\section{References}

[1] K. Aoyama, I. H. Koji, W. Takahashi, Weak convergence of an iterative sequence for accretive operators in Banach spaces, Fixed Point Theory Appl. (2006), Art. ID 35390, 13 pp.

[2] F. E. Browder, Convergenge theorem for sequence of nonlinear operator in Banach spaces, Math.Z. 100 (1967). 201-225. Vol. EVIII, part 2, 1976.

[3] I. Cioranescu, Geometry of Banach space, duality mapping and nonlinear problems, Kluwer, Dordrecht,1990.

[4] C. E. Chidume, Geometric Properties of Banach spaces and Nonlinear Iterations, Springer Verlag Series: Lecture Notes in Mathematics, Vol. 1965,(2009), ISBN 978-1-84882-189-7.

[5] K. Goebel, and W.A. Kirk, Topics in metric fixed poit theory, Cambridge Studies, in Advanced Mathemathics,. vo, 28, University Cambridge Press, Cambridge 1990.

[6] E. Hairer, S.P. Nrsett, G. Wanner, Solving Ordinary Differential Equations I: Nonstiff Problems, 2nd edn. Springer Series in Computational Mathematics. Springer, Berlin (1993).

[7] J.D. Hoffman, Numerical Methods for Engineers and Scientists, 2nd ed.; Marcel Dekker, Inc.: New York, NY, USA, 2001.

[8] Ke, Y. Ma, C., The generalized viscosity implicit rules of nonexpansive mappings in Hilbert spaces, Fixed Point Theory Appl. 2015, 2015, 190.

[9] T.C. Lim, H.K. Xu, Fixed point theorems for assymptoticaly nonexpansive mapping, Nonliear Anal 22(1994), no. 11, 13451355.

[10] W.R. Mann, Mean value methods in iteration, Proc. Amer. Math. Soc., 4 (1953) 506-510.

[11] P. E. Mainge, Strong convergence of projected subgradient methods for nonsmooth and nonstrictly convex minimization, Set-Valued Analysis, 16, 899-912 (2008).

[12] G. Marino, B. Scardamaglia, R. Zaccone, A general viscosity explicit midpoint rule for quasi-nonexpansive mappings, J.Nonlinear Convex Anal. 2017, 18, 137-148.

[13] A. Moudafi, Viscosity approximation methods for fixed point problems, J. Math. Anal. Appl. 241, 46-55 (2000).

[14] W. Nilsrakoo, S. Saejung, Weak and strong convergence theorems for countable Lipschitzian mappings and its applications, Nonlinear Anal. 69 (2008) 2695-2708.

[15] Z. Opial, Weak convergence of sequence of succecive approximation of nonexpansive mapping, Bull. Am. Math. Soc. 73 (1967), 591597.

[16] J. W. Peng, J.C. Yao, Strong convergence theorems of an iterative scheme based on extragradient method for mixed equilibruim problem and fixed point problems, Math. Com. Model. 49 (2009) 1816-1828.

[17] S. Reich, Weak convergence theorems for nonexpansive mappings in Banach spaces, Journal of Mathematical Analysis and Applications, vol. 67, no. 2, pp. 274276, 1979.

[18] C. Schneider, Analysis of the linearly implicit mid-point rule for differential-algebraic equations, Electron. Trans. Numer. Anal. 1, 1-10 (1993).

[19] T.M.M. Sow, N. DjittĖę, and C.E. Chidume, A path convergence theorem and construction of fixed points for nonexpansive mappings in certain Banach spaces, Carpathian J.Math.,32(2016),No.2,217-226,2016.

[20] K. Shimoji, W. Takahashi, Strong convergence to common fixed points of infinite nonexpansive mappings and applications, Taiwanese J. Math. 5 (2001).

[21] H. K. Xu, Inequalities in Banach spaces with applications, Nonlinear Anal. 16 (1991), no. 12, 1127-1138.

[22] H.K. Xu, Iterative algorithms for nonlinear operators, J. London Math. Soc. 66 (2002), no. 2, 240 - 256.

[23] H.K. Xu, M.A. Alghamdi, N. Shahzad, The viscosity technique for the implicit midpoint rule of nonexpansive mappings in Hilbert spaces, Fixed Point Theory Appl. 2015, 41 (2015).

[24] Y. Yao, H. Zhou, Y. C. Liou, Strong convergence of modified Krasnoselskii-Mann iterative algorithm for nonexpansive mappings, J. Math. Anal. Appl. Comput. 29 (2009) 383-389. 
[25] C. Zalinescu, On uniformly convex functions, J. Math. Anal. Appl. 95 (1983), 344-374. 